Revista de Economia Política, vol. 29, $n^{\circ} 3$ (115), pp. 232-251, julho-setembro/2009

\title{
Os efeitos da política educacional no crescimento econômico: teoria e estimativas dinâmicas em painel de dados
}

\author{
JOILSON DIAS \\ MARIA HELENA AMBRÓSIO DIAS \\ FERNANDINA FERNANDES DE LIMA*
}

Educational policies and economic growth: theory and dynamic panel data estimates. In this paper, we develop a theoretical model that considers a non-linear relationship between growth and level of education (human capital). Our econometric estimates demonstrated the causality running from human capital to GDP per capita with $U$ inverted shape. The level of education (human capital) that generates the maximum growth rate lies around 4.5 years. The foremost implication of this result is that States with level of education below this range should have as priority educational policies.

Keywords: Economic growth; education; panel dynamic estimation.

JEL Classificion: O15; C33.

O papel da educação na determinação da produtividade da economia iniciou-se com o trabalho de Schultz (1962) e Denison (1962). Ambos argumentaram a importância de se considerar o capital humano na função de produção agregada da economia. No entanto, a busca por uma explicação teórica do papel da educação no crescimento e desenvolvimento econômico iniciou-se efetivamente com o artigo de Uzawa (1965), que introduziu o setor educacional no modelo de crescimento econômico como forma de gerar crescimento contínuo e sustentado

\footnotetext{
* Respectivamente, Universidade Estadual de Maringá, Departamento de Economia, Maringá-PR. E-mail: jdias@uem.br; Departamento de Economia, Universidade Estadual de Maringá. E-mail: jdias@ uem.br; Aluna do Programa de Pós-Graduação em Economia, Universidade Estadual de Maringá, Maringá-PR. E-mail: fernandina@pop.com.br. Os autores agradecem imensamente o apoio financeiro recebido do $\mathrm{CNPq}$ - Conselho Nacional de Desenvolvimento Científico e Tecnológico e os comentários do parecerista anônimo. Submetido: Julho 2006: Aprovado: Fevereiro 2008.
} 
no longo prazo. O setor educacional tem papel preponderante, pois, é a fonte de aumentos da produtividade dos demais setores e, portanto, da economia. Apesar deste avanço, o modelo teórico proposto por Lucas (1988) foi o que consagrou a importância do capital humano em gerar crescimento sustentado. O autor demonstrou os efeitos das externalidades da acumulação de capital humano, sendo realizada no setor educacional sobre a produtividade da economia. Em seu modelo, o estoque de capital humano agregado gera um efeito de espraiamento (spill over), o que justifica os investimentos públicos em educação, devido ao ganho social advindo destes investimentos ${ }^{1}$.

Outro aspecto importante da acumulação de capital humano foi ressaltado por Romer (1990). Segundo este autor, o capital humano tem capacidade de gerar inovações (ideias) no setor de pesquisa e desenvolvimento. Como resultado, estas ideias produzem aumentos na produtividade agregada da economia, através da melhoria do coeficiente tecnológico dos insumos.

De forma resumida e do ponto de vista agregado, estes modelos preconizavam que a acumulação de capital humano (educação) melhora qualitativamente os capitais físicos e humanos. Além deste aspecto, aumenta seus coeficientes de produtividade, quando considerados em sua forma conjunta ${ }^{2}$.

Estes resultados teóricos foram testados inicialmente por Mankiw, Romer e Weil (1992). Na estimativa dos efeitos do capital humano sobre o crescimento econômico, os autores substituíram na equação os investimentos em educação por uma medida de investimento efetivo em educação. Assim, a quantidade de alunos matriculados no secundário foi utilizada como representante do investimento em educação realizado pelos países, portanto, foi considerada como proxy para o capital humano do país. O resultado foi de que o capital humano era o agente principal da causa dos aumentos de produtividade nas economias. No entanto, este resultado foi contestado por Benhabib e Spiegel (1994). Utilizando uma nova medida de capital humano, construída a partir dos diferentes níveis de escolaridade dos países, os autores não encontraram relação entre capital humano e crescimento econômico. Klenow e Rodriguez-Clare (1997) também encontraram um papel muito modesto para o capital humano na explicação das taxas de crescimento dos países. Este resultado modesto também aparece em artigo mais recente realizado por Wolf (2000). O autor apresentou um resultado similar para a educação secundária e terciária, inclusive em algumas especificações estas variáveis aparecem com sinal negativo. A única relação positiva encontrada, porém modesta, foi a da educação primária na explicação do crescimento da produtividade. No entanto, Hanushek e Kimko (2000) encontraram que a qualidade da educação é um importante elemento na explicação do crescimento econômico.

\footnotetext{
${ }^{1}$ Existe uma vasta literatura sobre estimativas de taxa de retorno social de investimento em educação, cf. Dias e Dias (2005).

${ }^{2}$ Para maiores detalhes desta ampla literatura, veja a revisão de literatura efetuada por Jones e Manuelli (1997a). Sobre o papel das fontes de crescimento, cf. Jones e Manuelli (1997b).
} 
A explicação para estes resultados contraditórios parece residir na forma da especificação econométrica estimada. Segundo Krueger e Lindahl (2001), estes resultados contraditórios são advindos da especificação da relação entre crescimento econômico e nível de educação. Todos os modelos mencionados, sem exceção, postulam uma relação linear. No entanto, em suas estimativas, Krueger e Lindahl (2001) obtiveram que a relação entre as duas variáveis é não-linear na forma de U invertido, conforme gráfico abaixo.

Figura 1: Relação entre taxa de crescimento e nível de educação

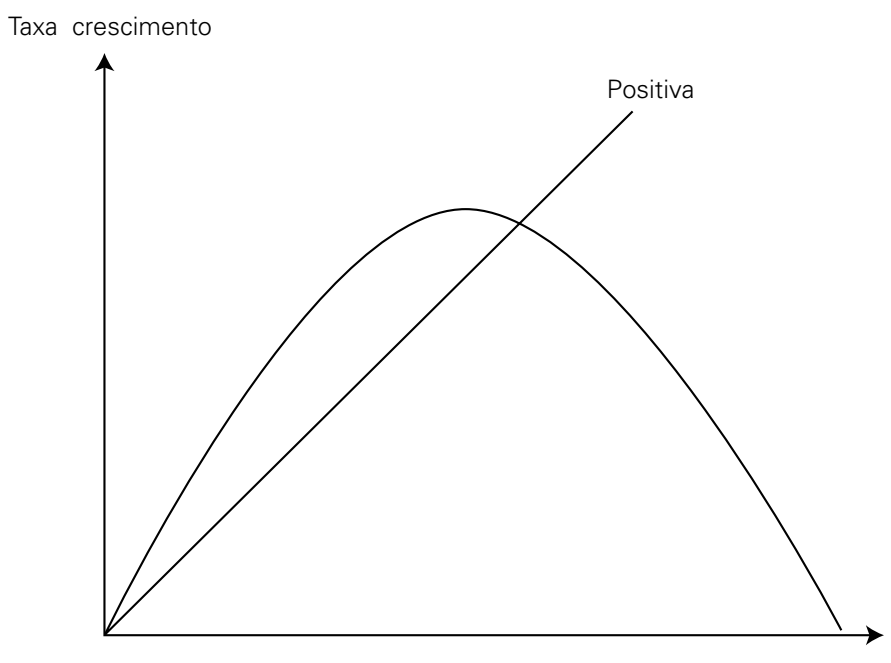

Fonte: Elaboração própria.

A implicação deste resultado empírico é extremamente interessante, pois os trabalhos que encontraram um resultado positivo, provavelmente estariam utilizando uma amostra em que o predomínio seria a primeira fase do gráfico, conforme indica sua linha positiva. Os demais trabalhos, com amostras mais amplas e estimativas lineares, não encontrariam qualquer relação entre crescimento econômico e nível de educação, em função de os pontos de influência positiva serem cancelados pelos que estão na descendência, influência negativa.

As estimativas utilizadas nos modelos econométricos têm como hipótese básica que a variável capital humano é independente, e, portanto, é a causa do crescimento econômico. No entanto, os questionamentos sobre a causalidade também estão presentes na literatura. Os trabalhos que encontraram causalidade entre capital humano e crescimento, mencionados anteriormente, foram contestados de forma mais contundente por Bils e Klenow (2000). Estes autores demonstraram que o crescimento esperado é capaz de gerar os mesmos resultados empíricos sobre a escolaridade. Esta causalidade reversa levou alguns pesquisadores a buscarem novos elementos como sendo a causa do crescimento econômico. Neste aspecto, destacou-se o trabalho de Hall e Jones (1999), que encontraram como fator explicativo para o desenvolvimento econômico, o capital social da economia, ou 
suas instituições. Outros trabalhos, como os de Beck, Levine e Loayza (2000), atestaram que as políticas econômicas, principais elementos resultantes das instituições, são as principais causas do crescimento ou da estagnação econômica. Easterly e Levine (2001) confirmaram estes resultados das políticas econômicas, no entanto, também encontraram papel importante para o nível de escolaridade (capital humano) no crescimento econômico. Em síntese, as políticas econômicas atuam de forma a amenizar ou exacerbar a acumulação de capital humano, causando assim, impacto no crescimento econômico de longo prazo.

O problema passou, então, a compreender o canal através do qual as políticas econômicas influenciam de forma direta ou indireta sobre a acumulação de capital humano e, por conseguinte, o crescimento econômico de longo prazo. Esta tarefa coube a Dias e McDermott (2006). Estes autores apresentaram um modelo teórico e efetuaram verificações empíricas. No modelo, a classe empresarial exerce importante papel no incentivo à acumulação de capital humano das demais classes e estas, por sua vez, auxiliam no crescimento econômico de longo prazo. Neste caso, os níveis de empreendedores das economias, sendo esta uma medida indireta de um tipo de capital humano especial, são uma das fontes do crescimento econômico de longo prazo. As políticas econômicas, que se referem a uma medida indireta da qualidade das instituições, fazem com que os níveis de empreendedores na economia sejam maiores ou menores, o que, por sua vez, causa maior ou menor acumulação de capital humano nas demais classes. Como resultado final, no longo prazo, tem-se um impacto no crescimento da economia ${ }^{3}$. O efeito feed back investigado, de que a melhoria do capital humano causaria um efeito positivo sobre a qualidade das políticas econômicas no longo prazo, demonstrou ser positivo, mas não suficientemente grande.

Assim, estas diferentes medidas de capital social e/ou políticas econômicas resultantes das instituições, passaram a ser referidas simplesmente como instituições do país e, portanto, à luz dos fatos, precederiam em importância em relação à acumulação dos fatores de produção, em especial do capital humano. Portanto, a pergunta natural seria a de investigar até que ponto as instituições são a causa ou a consequência do capital humano. Glaeser, La Porta, Lopez-de-Silanes e Shleifer (2004) efetuaram tal pergunta. A principal hipótese deste questionamento passa a ser, então, se o capital humano (escolaridade) precede ou não as instituições. Ou seja, primeiro a população se educa e depois constrói as instituições que afetam o crescimento econômico ou vice-versa. Como resultado, os autores obtiveram que os níveis iniciais de educação são fortes determinantes de melhores instituições no futuro. Este resultado é consistente com o fato de que, mesmo em um horizonte curto de cinco anos, o capital humano é capaz de produzir melhores instituições subsequentemente e, consequentemente, crescimento econômico. Em síntese, em primeiro lugar vem a educação, e esta causa uma melhoria nas instituições, que,

\footnotetext{
${ }^{3}$ A classe empresarial determinaria a taxa de retorno da educação na economia ao criar um mercado para o capital humano das demais classes.
} 
por sua vez, pode resultar em um incentivo adicional para a acumulação de capital humano, sendo este o elemento gerador do crescimento econômico de longo prazo. A condição primeira é a que será verificada para os estados brasileiros e, em especial, se o horizonte de cinco anos de acumulação de educação é suficiente para causar um aumento no crescimento da produtividade no longo prazo.

O artigo está distribuído da seguinte forma: primeira seção, que é esta introdução; segunda seção, na qual é apresentado o modelo teórico de crescimento econômico de longo prazo, cujo resultado principal é a não-linearidade entre capital humano e crescimento econômico; terceira seção, em que são realizadas estimativas econométricas, e para isso foram estimados dois modelos, efeitos fixos e randômicos, para utilizá-los como base. A seguir, o modelo dinâmico é estimado, o que permite resolver o problema de causalidade simultânea das variáveis; e, por último, são apresentadas as conclusões.

\section{MODELO TEÓRICO}

O modelo teórico a ser desenvolvido é uma combinação dos pressupostos de linearidade da função de produção em relação ao fator capital físico de Rebelo (1991), com a importância do capital humano de Lucas (1988). No entanto, o papel do capital humano neste modelo está em tornar o ativo capital físico mais produtivo, portanto, aqui, os preceitos do modelo de Romer (1990) são seguidos. O papel do setor público neste modelo é o de atuar de forma eficiente na transferência de recursos do setor produtor de bens para o setor encarregado da acumulação de acumular capital humano, ou seja, todo o imposto arrecadado é convertido na acumulação de capital humano. O modelo contempla ainda os seguintes aspectos: i) o setor produtor de bens de consumo e ii) o setor capital humano, que tem como função a de aumentar a produtividade dos agentes econômicos.

A função de produção linear proposta por Rebelo (1991) pode ser apresentada da seguinte forma:

$$
\mathrm{y}=(1-\tau) \mathrm{Ak}
$$

$\mathrm{Na}$ qual y é a produção por trabalhador; A é o nível tecnológico (ou eficiência do fator k); k é o estoque de capital físico; e $\tau$ é o nível de tributos na economia que é destinado ao setor de acumulação de capital humano. Como se pode observar esta especificação contempla somente o efeito da acumulação de capital físico que gera aumentos na produtividade por trabalhador. Para contemplar o papel do capital humano, segundo Lucas (1988) e Romer (1990), a função acima será especificada da seguinte forma:

$$
\mathrm{y}=(1-\tau) \mathrm{A}(\mathrm{h}) \mathrm{k}
$$

sendo que $\mathrm{A}(\mathrm{h})$ informa o nível de tecnologia ou eficiência do capital físico por trabalhador que está associado ao capital humano do trabalhador (h), cujo es- 
toque agregado da economia é simplesmente $\mathrm{H}=\mathrm{hn}$, sendo n o número total de trabalhadores.

Na equação (2), quanto maior o nível médio do conhecimento (escolaridade) h, maior é a produção de tecnologia e, por conseguinte, a produtividade. Este efeito gera benefícios agregados para a economia, permitindo que a decisão de acumulação de $\mathrm{k}$, pelos indivíduos, se torne independente das demais variáveis. Como será visto a seguir, h é acumulado por um setor educacional que recebe transferências governamentais obtidas através de impostos sobre o setor produtivo. O nível de taxação dos impostos da economia é escolhido, de tal forma, que os benefícios resultantes sejam iguais aos sacrifícios impostos na produção. Esta forma exógena de decisão evita que seja incorporado dentro do modelo a atuação do governo.

Neste modelo, o comportamento dos indivíduos é descrito por uma função de bem-estar que, por simplicidade, assume a seguinte forma:

$$
\mathrm{u}(\mathrm{c})=\int_{0}^{\infty} \frac{c^{1-\sigma}-1}{1-\sigma} e^{-p t} d t \text { para } \sigma \neq 1
$$

A função (3) depende do consumo per capita (c) e a elasticidade de substituição intertemporal é constante e igual a $(1 / \sigma)$. O consumidor representativo desconta sua utilidade futura a uma taxa constante de preferência $\rho$. A restrição é dada pela seguinte equação:

$$
\dot{\mathrm{k}}=(1-\tau) \mathrm{A}(\mathrm{h}) \mathrm{k}-\mathrm{c}
$$

$\mathrm{Na}$ função acima $\dot{\mathrm{k}}$ representa a variação do estoque de capital. De forma simplificada esta função informa que o que os bens não consumidos são transformados em estoque de capital no período seguinte. Para simplificar, assume-se que a população e os trabalhadores crescem a uma taxa constante e igual a $\mathrm{n}$.

A acumulação de capital humano proposta por Lucas (1988) é a seguinte $\dot{\mathrm{h}}=\delta(1-\mathrm{u}) \mathrm{h}$, sendo que $(1-\mathrm{u})$ representa o nível de esforço do capital humano $(\mathrm{h})$ existente em direção a acumulação de capital humano ( $\dot{\mathrm{h}}$ ). No modelo aqui especificado, o nível de esforço em direção à acumulação de capital humano é equivalente ao nível de taxação do produto que é investido em capital humano, $\tau$. Portanto, o investimento em capital humano equivale a percentagem dos tributos arrecadados como proporção do produto total da economia. A especificação da acumulação de capital humano é a seguinte:

$$
\dot{\mathrm{h}}=\delta \tau \mathrm{h}
$$

A taxa de crescimento do capital humano está expressa em termos da taxa do esforço da economia, onde $\delta$ é a medida de eficiência do setor educacional. Se $\delta=0$, significa que a eficiência de conversão de tributos em crescimento do capital humano é zero; se $\delta>1$, significa que a taxa de crescimento do capital humano é superior à taxa de sacrifício sendo imposta aos consumidores.

O Hamiltoniano das equações (3), (4) e (5) é o seguinte:

$$
\mathrm{H}=\frac{C^{1-\sigma}}{1-\sigma}+\lambda_{1}[(1-\tau) \mathrm{Ak}-\mathrm{c}]+\lambda_{2}(\delta \text { th })
$$


O Hamiltoniano acima está expresso em termos de valor presente, onde a taxa de desconto no tempo é $\rho$. É importante notar que $\lambda_{1}$ e $\lambda_{2}$ representam os preços dos capitais físicos e humanos, em termos de utilidades, o chamado preço sombra. A maximização da função acima equivale ao seguinte ${ }^{4}$ :

$$
\begin{aligned}
& \frac{\partial \mathrm{H}}{\partial \mathrm{c}}=0 \Rightarrow \mathrm{c}^{-\sigma}=\lambda_{1} \\
& \dot{\lambda}-\rho \lambda_{1}=-\frac{\partial \mathrm{H}}{\partial \mathrm{k}} \\
& \dot{\lambda}_{2}-\rho \lambda_{2}=-\frac{\partial \mathrm{H}}{\partial \mathrm{h}}
\end{aligned}
$$

As condições de transversalidade são as seguintes:

$$
\begin{aligned}
& \lim _{t \rightarrow \infty} k(t) \lambda_{l}(t) e^{-\rho t}=0 \\
& \lim _{t \rightarrow \infty} h(t) \lambda_{2}(t) e^{-\rho t}=0
\end{aligned}
$$

O resultado das equações (9) e (10) são as seguintes:

$$
\begin{aligned}
& \frac{\dot{\lambda}_{1}}{\lambda_{1}}=-[(1-\tau) A+\rho] \\
& \frac{\dot{\lambda}_{2}}{\lambda_{2}}=-\frac{\lambda_{1}}{\lambda_{2}}\left[(1-\tau) \frac{\partial A}{\partial h} k\right]-\tau \delta+\rho
\end{aligned}
$$

O objetivo é solucionar o modelo sob a condição de crescimento equilibrado. Para tanto, a regra utilizada por Lucas (1988) é utilizada para contemplar a participação do governo na decisão de investir em educação. Assim, a decisão segue a regra de que o valor marginal arrecadado em tributos deva ser igual ao valor marginal produzido de capital humano, ou seja

$$
\tau \lambda_{1} A k=\tau \lambda_{2} \delta h
$$

A equação acima é importante, porque para valores de $\tau \in(0,1)$ os valores marginais dos sacrifícios entre a acumulação de capital físico e humano são equalizados. Ou seja, gera um processo sustentado de acumulação de capitais físico e humano no tempo. A implicação desta escolha do ponto de vista econômico pode ser visualizada quando solucionamos para as funções de crescimento do consumo, capital físico e humano. A função de crescimento do consumo é obtida da combinação das equações (7) e (12), enquanto que a de crescimento do capital físico é obtida da combinação da condição de transversalidade (10) com a equação (12) e a função de crescimento do capital humano é simplesmente a equação (5).

\footnotetext{
${ }^{4}$ Apesar de o Hamiltoniano possuir duas variáveis de escolha $(c, \tau)$, o processo de maximização em relação a variável $\tau$ não se aplica uma vez que a função Hamiltoniana é linear em relação a mesma. Portanto, a sua escolha será feita visando obedecer às condições econômicas, mas fora do ela de maximização.
} 


$$
\begin{aligned}
\frac{\dot{c}}{c} & =\frac{1}{\sigma}[(1-\tau) A-\rho] \\
\frac{\dot{k}}{k} & =[(1-\tau) A] \\
\frac{\dot{h}}{h} & =\delta \tau
\end{aligned}
$$

Em resumo, a escolha do valor de $\tau$ gera sacrifícios na economia em termos da taxa de crescimento do consumo e dos investimentos em capital físico. Estes sacrifícios são compensados pelo crescimento do setor que acumula capital humano. Portanto, uma solução plausível a ser desenvolvida visa manter esta condição de equilíbrio econômico no longo prazo. Assim, basta tomar o logaritmo da equação (14) e diferenciar com relação ao tempo para obter a relação entre as taxas de crescimento das variáveis e seus preços sombra de crescimento equilibrado. Lembrando que $\delta$ e $\tau$ são constantes, tem-se:

$$
\frac{\dot{\lambda}_{1}}{\lambda_{1}}+\frac{\dot{A}}{A}+\frac{\dot{k}}{k}=\frac{\dot{\lambda}_{2}}{\lambda_{2}}+\frac{\dot{h}}{h}
$$

A solução final requer que primeiro seja resolvida a equação (14) para a razão dos preços sombra dos capitais em seguida substituída na equação (13). Este resultado, juntamente com as equações (5) e (12) são substituídos na equação (18). Após as devidas simplificações, obtem-se o seguinte resultado:

$$
\frac{\dot{A}}{A}+\frac{\dot{k}}{k}=(1-\tau) A-(1-\tau) \frac{\delta A_{h} h}{A}
$$

$\mathrm{Na}$ equação acima, o lado esquerdo é a taxa de crescimento da economia através de seus insumos e $A_{h}=\frac{\partial A}{\partial h}$. Para demonstrar que o lado esquerdo é igual à taxa de crescimento da economia, utiliza-se a equação (1). Tirando o logaritmo e diferenciando com relação ao tempo, tem-se que:

$$
g_{y}=\frac{\dot{y}}{y}=\frac{\dot{A}}{A}+\frac{\dot{k}}{k}
$$

Portanto, utilizando a equação acima, pode-se reescrever a equação (19) da seguinte forma:

$$
g_{y}=(1-\tau)\left[A-\frac{\delta A_{h} h}{A}\right]
$$

Como a variável A é uma função do capital humano, tem-se que a taxa de crescimento do produto per capita depende única e exclusivamente do nível de capital humano da economia. Para demonstrar que a equação (18) está em acordo com os resultados obtidos por Krueger e Lindahl (2001), basta admitir que $A=\omega \mathrm{h}$, onde $\omega>0$. Ou seja, o nível de tecnologia é uma função linear do capital humano. Portanto, a equação acima se torna a seguinte:

$$
g_{y}=-(1-\tau) \delta+(1-\tau) \omega h
$$


Para concluir, assume-se que a função capital humano é quadrática no nível de escolaridade (E), ou seja: $h=a E-b^{2}$, onde $a$ e $b>0$ são constantes a serem obtidas em estimativas para o Brasil. Substituindo a definição de capital humano na equação (22), tem-se que:

$$
g_{y}=-(1-\tau) \delta+(1-\tau) \omega a E-(1-\tau) \omega b E^{2}
$$

Em outras palavras, a taxa de crescimento do produto per capita de longo prazo depende do esforço efetuado na acumulação do nível de escolaridade (E). $\mathrm{Na}$ equação acima, pode-se observar que o primeiro termo do lado direito é negativo [(1- $\tau) \delta]$, portanto, o modelo tem como resultado, o fato de que deva ocorrer um sacrifício inicial de crescimento econômico, para que se obtenha, finalmente, o efeito do nível de escolaridade sobre a taxa de crescimento ${ }^{5}$. Este resultado pode explicar, em parte, os resultados contraditórios, em especial quando na amostra encontramse países com baixo e alto nível de escolaridade, como obtidos por Easterly (2001). Neste caso, há uma influência negativa dos países de baixo nível de escolaridade com os efeitos decrescentes dos países com alto nível de escolaridade.

Em resumo, a taxa de crescimento somente é influenciada pela escolaridade após a economia ter alcançado um determinado nível de capital humano, aqui representado por uma função não-linear em relação à escolaridade. A partir deste ponto, a economia começa a colher os benefícios da sua acumulação de capital humano. Para uma melhor compreensão, a equação (2) foi simulada para os seguintes parâmetros: $\tau=0,20, \delta=0,03, \omega \mathrm{a}=0,021$ e $\omega \mathrm{b}=0,002$.

Figura 2: Resultado da simulação: crescimento e nível de escolaridade

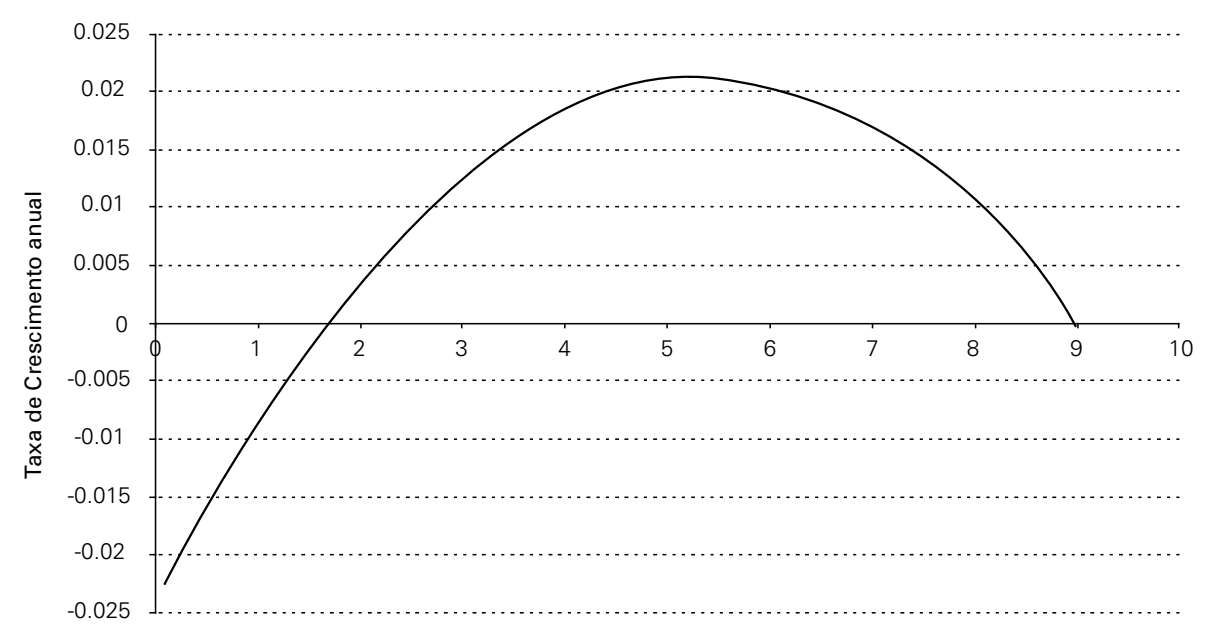

Nível de Escolaridade

\footnotetext{
${ }^{5}$ Este resultado é compatível com os obtidos empiricamente por Dias e Dias (2007) para os estados brasileiros. Ou seja, os gastos públicos com educação exercem efeitos negativos nas taxas de crescimento dos estados brasileiros.
} 
Para estes valores, a taxa de crescimento do produto per capita somente recebe influência positiva quando o nível de escolaridade ultrapassa 1,7 anos. A partir deste valor, até aproximadamente 5,0 anos de escolaridade, em média, existe uma forte influência na taxa de crescimento do nível de escolaridade. Cabe ressaltar que, a simulação acima, não determina o efeito de causalidade, portanto há somente uma relação entre as duas variáveis. Para estimar a relação causal, será especificada novamente a equação (23) da seguinte forma:

$$
g_{y}=\beta_{0}+\beta_{1} E+\beta_{2} E^{2}+e
$$

Sendo $\beta_{0}=-(1-\tau) \delta ; \beta_{1}=(1-\tau) a \omega ;$ e $\beta_{2}=-(1-\tau) \omega b$; e, $e$, os erros de estimação da regressão. Na próxima seção, discute-se a aplicação do método de estimação dinâmica relacionando-a com a questão da causalidade entre capital humano e crescimento econômico.

\section{ESTIMATIVAS ECONOMÉTRICAS}

\section{Introdução}

Antes de um aprofundamento na questão do método econométrico a ser empregado, será feita uma breve discussão sobre os dados. Os dados referem-se aos estados brasileiros no período de 1985-2000. Considerando os 26 estados e o período de 16 anos, para os quais temos os dados completos, temos um total de 416 observações a serem utilizadas. Um detalhe importante é o fato de que o estado de Tocantins foi desconsiderado por apresentar dados somente a partir da década de 1990. A fonte dos dados é o IPEA - Instituto de Pesquisa Econômica e Aplicada ${ }^{6}$. Basicamente, são utilizadas três variáveis, quais sejam:

a) Taxa de crescimento do Produto Interno Bruto (PIB) per capita dos estados, $\mathrm{g}_{\mathrm{y}}$;

b) Média da escolaridade das pessoas com 25 anos ou mais dos estados. Valor este medido em número de anos que frequentaram a escola;

c) Número médio de horas trabalhadas por semana nos estados. Esta variável é utilizada como proxy da instituição mercado de trabalho de cada estado.

A Tabela 1 apresenta as variáveis dos estados de forma sumarizada.

\footnotetext{
${ }^{6}$ Disponível em: www.ipeadata.gov.br.
} 
Tabela 1: Sumário dos dados dos estados brasileiros: período 1985-2000

\begin{tabular}{|c|c|c|c|c|c|c|}
\hline Variável & & Média & Desvio-padrão & Mínimo & Máximo & $\begin{array}{c}\text { Observa- } \\
\text { ções }\end{array}$ \\
\hline \multicolumn{7}{|l|}{ gy } \\
\hline & Total & 0,0358 & 0,0761 & $-0,1787$ & 0,2867 & $N=416$ \\
\hline & Entre & & 0,0135 & 0,0039 & 0,0643 & $N=26$ \\
\hline & Dentro & & 0,0751 & $-0,1705$ & 0,2853 & $\mathrm{~T}=16$ \\
\hline \multicolumn{7}{|l|}{$\mathrm{n}$} \\
\hline & Total & 41,5 & 2,9 & 32,3 & 48,9 & $N=416$ \\
\hline & Entre & & 1,9 & 36,7 & 44,3 & $N=26$ \\
\hline & Dentro & & 2,2 & 32,4 & 47,6 & $\mathrm{~T}=16$ \\
\hline \multicolumn{7}{|l|}{$\mathrm{h}$} \\
\hline & Total & 4,83 & 1,23 & 2,05 & 8,17 & $N=416$ \\
\hline & Entre & & 1,15 & 2,90 & 7,54 & $N=26$ \\
\hline & Dentro & & 0,49 & 3,47 & 5,87 & $\mathrm{~T}=16$ \\
\hline \multicolumn{7}{|l|}{ h2 } \\
\hline & Total & 24,86 & 12,20 & 4,22 & 66,86 & $N=416$ \\
\hline & Entre & & 11,46 & 8,92 & 57,14 & $N=26$ \\
\hline & Dentro & & 4,72 & 11,67 & 36,63 & $\mathrm{~T}=16$ \\
\hline
\end{tabular}

Fonte: IPEA.

Apesar de ser de fácil compreensão a tabela acima, far-se-á a leitura da primeira variável, que é a taxa de crescimento do PIB per capita dos estados no período. A média no período dos estados foi de 3,58\%, com um desvio-padrão de $7,61 \%$. Os valores máximos e mínimos refletem a existência de alguma discrepância, quer seja de problemas de amostra, ou de efeitos não observados. A menor taxa de crescimento verificada nos estados foi de $-17,87 \%$, sendo que a maior taxa registrada foi de $28,67 \%$. Estas taxas certamente apresentam-se como potenciais outliers $^{7}$. Nas estimativas a serem realizadas, os efeitos destes outliers tendem a ser minimizados através da utilização de variância específica de painéis.

\section{Modelo de efeitos fixos e/ou randômicos}

Nesta seção, o objetivo é testar o modelo mais adequado e efetuar a estimativa dinâmica da equação (24). Portanto, inicialmente serão feitos os testes de escolha entre os modelos de efeitos fixos e os modelos de efeitos randômicos. O passo seguinte apresenta os testes habituais de autocorrelação e heterocedasticidade. $\mathrm{O}$ modelo mais adequado, de acordo com os testes, será então utilizado como base para as estimativas dinâmicas.

A especificação do modelo econométrico para a equação (24) é a seguinte:

\footnotetext{
${ }^{7}$ Testes realizados visando à análise de outliers corroboram com a hipótese de sua existência.
} 


$$
\begin{aligned}
& y_{i, t}=x_{i, t} \beta+\varepsilon_{i, t}, \\
& \varepsilon_{i, t}=\alpha_{i}+\eta_{i, t}
\end{aligned}
$$

No qual $\mathrm{y}_{\mathrm{i}, \mathrm{t}}$ representa a variável dependente com $\mathrm{i}=1, \ldots, 26$ e $\mathrm{t}=1, \ldots, 16 ; \mathrm{x}_{\mathrm{i}, \mathrm{t}}$ representa a matriz de variáveis independentes; e, $\varepsilon_{i t}$ é o vetor que contempla os efeitos fixos. As hipóteses iniciais a serem estimadas sobre o modelo acima são as seguintes:

$$
\begin{aligned}
& E\left(\alpha_{i}\right)=0, \\
& E\left(\eta_{i, t}\right)=0 \\
& E\left(\alpha_{i} \alpha_{i}\right)=\sigma_{\alpha} \\
& E\left(\eta \eta^{\prime}\right)=\sigma_{n}^{2} I_{n t} \\
& E\left(\alpha_{i} \eta_{i, t}\right)=0 \\
& E\left(\alpha_{i} \alpha_{j}\right)=0 ; \text { se } i \neq j .
\end{aligned}
$$

As hipóteses (27), (28) e (31) e a condição de que $\mathrm{E}\left(\alpha_{\mathrm{i}} \mathrm{X}_{\mathrm{i}, \mathrm{j}}\right)=0$ estabelecem que o modelo é de efeitos randômicos. Caso não sejam verificadas, o modelo estabele-

\begin{tabular}{|c|c|c|}
\hline $\begin{array}{c}\text { Variáveis } \\
\text { (1) }\end{array}$ & $\begin{array}{l}\text { Fixo } \\
(2)\end{array}$ & $\begin{array}{c}\text { Randômico } \\
\text { (3) }\end{array}$ \\
\hline $\mathrm{H}$ & $\begin{array}{l}-0,0238 \\
(-0,0313)\end{array}$ & $\begin{array}{c}- \\
-0,00349 \\
(0,0186)\end{array}$ \\
\hline \multirow[t]{2}{*}{ h2 } & 0,00185 & 0,00028 \\
\hline & $(0,0033)$ & $(0,00189)$ \\
\hline \multirow{2}{*}{ Constante } & 0,1048 & 0,0475 \\
\hline & $(0,074)$ & $(0,0445)$ \\
\hline $\mathrm{R} 2$ & 0,006 & 0,006 \\
\hline$\sigma_{\alpha}$ & 0,015 & 0 \\
\hline$\sigma_{\eta}$ & 0,077 & 0,077 \\
\hline$F(25,388)$ All $(\alpha i)=0$ & rion & \\
\hline N & 416 & 416 \\
\hline
\end{tabular}
cido é de efeitos fixos. Ambos os modelos pressupõem a exogeneidade estrita como questão essencial, que em termos dos erros pressupõem que obedecem à seguinte condição: $E\left(\eta_{\mathrm{i}, \mathrm{t}}, \mathrm{x}_{\mathrm{i} 1}, \ldots, \mathrm{x}_{\mathrm{iT}}, \alpha_{\mathrm{i}}\right)=0$. Assim, primeiro estima-se os dois modelos sob as condições de efeitos fixos e randômicos e, em seguida, são efetuados os testes de definição. A Tabela 2 apresenta os resultados.

Tabela 2: Os modelos de efeitos fixos e randômicos

Explicando a Taxa de Crescimento do PIB Per Capita

Como é possível observar, em ambos os modelos as variáveis se apresentam como sendo não significativas, isto é, com poder de explicação muito baixo. A não-significância dos parâmetros, bem como o baixo poder de explicação, pode 
estar associada, como poderá ser visto mais adiante, aos problemas de heterocedasticidade e autocorrelação. A priori, a única informação relevante nas regressões acima é que o teste $\mathrm{F}$, na coluna (1), indica que o modelo mais adequado é o de efeitos randômicos.

A seguir foi aplicado o teste de especificação do modelo proposto por Hausman (1978). Neste teste, os coeficientes dos modelos de efeitos fixos e randômicos são averiguados quanto às suas igualdades estatísticas. A igualdade observada indica que a melhor especificação é a randômica, pois considera a condição de ortogonalidade entre os coeficientes fixos e as variáveis independentes. Indiretamente, este teste verifica se a hipótese da equação (24) é realmente válida. O resultado do teste de Qui-Quadrado, $\chi^{2}=1,02$, apresenta uma probabilidade de aceitação de igualdade dos coeficientes de $60,03 \%$. Esta alta probabilidade confirma o modelo de efeitos randômicos como o mais indicado. No entanto, este teste sofre da influência da autocorrelação, quando presente. Assim, visando considerar este aspecto, é efetuado o conjunto de testes de Máxima Verossimilhança (MV) para efeitos randômicos, proposto por Breusch e Pagan (1980); e, testes para efeitos randômicos em conjunto com a autocorrelação, proposto por Baltagi e Li (1995). A Tabela 3 apresenta o resumo dos testes.

Tabela 3: Testes randômico e correlação serial

\begin{tabular}{c|c|c}
\hline $\begin{array}{c}\text { Testes } \\
\text { (1) }\end{array}$ & Resultado do Teste & $\begin{array}{c}\text { Probabilidade H0 } \\
\text { (2) }\end{array}$ \\
\hline Efeitos Randômicos & & 0,98 \\
MV: $\operatorname{Var}(\alpha i)=0$ & $-1,89$ & 0,93 \\
MVA: $\operatorname{Var}(\alpha i)=0$ & $-1,47$ & \\
Correlação Serial & & 0,14 \\
MV: $\rho=0$ & 2,15 & 0,39 \\
MVA: $\rho=0$ & 0,73 & \\
Teste Conjunto & & 0,12 \\
MV: $\operatorname{Var}(\alpha i)=0$ e $\rho=0$ & 4,31 & \\
\hline
\end{tabular}

Os testes de efeitos randômicos, tanto o de máxima verossimilhança sem correção para a autocorrelação (MV) como o que considera a correção para autocorrelação (MVA), informam que o modelo de efeito randômico é o que deve ser considerado. Os testes de autocorrelação serial, apesar de baixos, indicam que sua presença é comum a todos os painéis, portanto, se existe autocorrelação, esta deve ser específica. O teste conjunto que verifica a presença do efeito randômico, e sem autocorrelação, é ligeiramente superior ao nível mínimo aceitável de $10 \%$.

Para dar maior robustez aos nossos testes, um teste adicional de autocorrelação proposto por Woodridge (2002) foi efetuado. O resultado do teste $\mathrm{F}(1,25)=$ 0,134 indica uma probabilidade de $71,79 \%$ de aceitação da não existência de autocorrelação de primeira ordem. 
A seguir, realizou-se o teste de heterocedasticidade, proposto por Greene (2000). Este teste verifica a igualdade das variâncias entre os painéis. O resultado da estatística $\chi^{2}=122,58$ informa que a probabilidade dos painéis terem a mesma variância é nula. Portanto, é detectada a presença da heterocedasticidade nos dados.

Este conjunto de testes nos aponta para um modelo de efeito randômico que deve ser corrigido para heterocedasticidade. Além deste aspecto, deve-se considerar o potencial de autocorrelação específica de painéis nas estimativas. Para isso, são feitas quatro estimativas usando dois métodos diferentes. O primeiro método, proposto por Prais-Winstem, descrito em Judge et al. (1985), estima o modelo usando Mínimos Quadrados Ordinários (MQO-PW), que, além de corrigir o problema de heterocedasticidade, também efetua duas correções para a autocorrelação. A primeira correção para correlação serial utiliza um coeficiente comum a todas as observações, enquanto a segunda corrige cada painel pelo seu respectivo coeficiente de correlação serial. O segundo método proposto por Corchrane e Orcutt, descrito em Judge et al. (1985), emprega Mínimos Quadrados Generalizados Factíveis (MQGF) e, como o anterior, corrige os problemas de heterocedasticidade e das autocorrelações. Os resultados destes métodos podem ser analisados na Tabela 4.

Tabela 4: Os métodos MQO-PW e MQGF explicando a taxa de crescimento do PIB Per Capita

\begin{tabular}{c|c|c|c|c}
\hline $\begin{array}{c}\text { Variáveis } \\
(1)\end{array}$ & $\begin{array}{c}\text { MOO-PW } \\
(2)\end{array}$ & $\begin{array}{c}\text { MOO-PW } \\
(3)\end{array}$ & $\begin{array}{c}\text { MOGF } \\
(4)\end{array}$ & $\begin{array}{c}\text { MOGF } \\
(5)\end{array}$ \\
\hline$h$ & 0,016 & 0,017 & 0,018 & 0,019 \\
& $(0,003)^{*}$ & $(0,003)^{*}$ & $(0,003)^{*}$ & $(0,003)^{*}$ \\
$h^{2}$ & $-0,0016$ & $-0,0019$ & $-0,0022$ & $-0,0024$ \\
$R^{2}$ & $(0,0006)^{*}$ & $(0,0006)^{*}$ & $(0,0005)^{*}$ & $(0,0005)^{*}$ \\
$\chi^{2}$ & 0,20 & 0,23 & - & - \\
& 102,78 & 129,58 & 130,86 & 163,13 \\
$\rho$ & $(0,00)$ & $(0,00)$ & $(0,00)$ & $(0,00)$ \\
$N$ & $-0,06$ & $-0,03, \ldots, 0,24$ & $-0,06$ & $-0,03, \ldots, 0,24$ \\
& 416 & 416 & 416 & 416 \\
\hline
\end{tabular}

* Indica nível de significância de 1\%. Os desvios-padrão entre parênteses são robustos aos problemas de heterocedasticidade e autocorrelação entre painéis.

Os modelos foram estimados utilizando a mesma especificação, mas com correção de autocorrelação diferente. Nas colunas (2) e (4) da Tabela 4 a autocorrelação é única para todos os painéis, portanto, aparece somente um valor para $\rho=-$ 0,06 . Nas colunas (3) e (5) da mesma tabela, as estimativas consideram um coeficiente de autocorrelação para cada painel, sendo que os valores do coeficiente se situam no intervalo $\rho=-0,03$ e $\rho=-0,24$. Os resultados dos coeficientes são estatisticamente idênticos, sendo que oscilam dentro do intervalo de seus desvios padrões. O coeficiente de determinação $\left(\mathrm{R}^{2}\right)$ entre $20 \%$ e $23 \%$ é considerado como 
muito bom e compatível com os obtidos na literatura ${ }^{8}$. As estimativas são significantes e confirmam a não-linearidade entre taxa de crescimento e nível de escolaridade. Os parâmetros estimados são muito próximos daqueles utilizados para simular a Figura 1, sendo que a única diferença reside na constante. As estimativas da constante apresentaram-se como sendo insignificantes em todas as especificações, portanto foi desconsiderada. Similar resultado sobre a constante foi encontrado por Krueger e Lindahl (2001). A explicação apresentada para o fato é bastante simples. Como a amostra utilizada é truncada para um nível de escolaridade mínimo de 2,05 anos, não se observam estados com um nível de escolaridade iniciando a partir do zero. Isto equivale a deslocar o eixo vertical a partir do ponto de início de importância da escolaridade, que, na simulação, foi de 1,7 ano. Ou seja, este deslocamento do eixo vertical provocado pelos dados faz com que a constante estimada seja zero.

O significado econômico da constante é muito importante. Quando o nível de escolaridade é abaixo do mínimo, como neste modelo, geram ganhos inferiores ao custo do sacrifício imputado. Neste caso, não há incentivos à acumulação de capital humano. Para suplantar estas perdas iniciais, faz-se necessário a presença de uma política contínua de acumulação de capital humano até ultrapassar o valor mínimo. Caso esta política não seja feita de forma contínua até ultrapassar este limite mínimo, a economia pode ficar presa numa armadilha de crescimento esperado negativo, dependendo de políticas exógenas à acumulação de capital humano.

\section{Modelo dinâmico}

As estimativas dinâmicas consistem em verificar se o nível de escolaridade precede o crescimento econômico, conforme modelo teórico. No entanto, o objetivo não é apenas este, mas também o de verificar o efeito feed back do próprio crescimento. O método econométrico dinâmico (MED), que permite testar estas hipóteses, foi construído por Arellano e Bond (1991), Arellano e Bover (1995) e Blundell e Bond (1998). O modelo proposto é uma ligeira modificação da equação (23), pois considera os efeitos do próprio crescimento passado sobre o crescimento atual em nível e diferença, portanto, um sistema de equações:

$$
\begin{aligned}
& y_{i, t}=\alpha y_{i, t-1}+x_{i, t} \beta+\varepsilon_{i, t} \\
& y_{i, t}-y_{i, t-1}=\alpha\left(y_{i, t-1}-y_{i, t-2}\right)+\left(x_{i, t}-x_{i, t-1}\right) \beta+\left(\varepsilon_{i, t}-\varepsilon_{i, t-1}\right)
\end{aligned}
$$

Se o crescimento no período anterior reflete todas as variáveis que o influenciaram, então, ao considerar a variável dependente defasada, como variável explicativa, tem-se um modelo dinâmico capaz de capturar os efeitos do período anterior. Assim, os efeitos remanescentes sobre a taxa de crescimento econômico devem estar associados ao capital humano, proposta pelo modelo.

\footnotetext{
${ }^{8}$ Veja por exemplo Hall e Jones (1999).
} 
A estimativa do sistema de equações (33) e (34) utiliza como variáveis instrumentos para equação em nível (30) as diferenças das variáveis explicativas; e, para a equação em diferença, as variáveis em níveis. Para ser compatível com a proposta de Glaeser et al. (2004), os instrumentos serão defasados em cinco anos. É importante lembrar, contudo, que Krueger e Lindahl (2001) utilizaram as defasagens de 10 e 20 anos. Neste caso, testa-se se a escolaridade precede o crescimento econômico nos estados brasileiros, evitando o chamado efeito de causalidade reversa. Os resultados obtidos através do método de momentos generalizados estão na Tabela 5 .

Nos resultados da tabela abaixo, tem-se que as variáveis instrumentais da escolaridade, tanto em nível quanto em diferença, são as próprias variáveis defasadas em cinco períodos. Portanto, as equações são estimadas simultaneamente sob a condição de que os movimentos nas variáveis escolaridade e escolaridade ao quadrado precedem o crescimento econômico em cinco anos. Os resultados dos coeficientes são altamente significativos e compatíveis com os resultados da Tabela 4, obtidos sob a condição de ortogonalidade das variáveis para o mesmo período.

Tabela: O método econométrico dinâmico explicando a taxa de crescimento do PIB Per Capita

\begin{tabular}{c|c|c}
\hline $\begin{array}{c}\text { Variáveis } \\
(1)\end{array}$ & $\begin{array}{c}\text { MED } \\
(2)\end{array}$ & $\begin{array}{c}\text { MED } \\
(3)\end{array}$ \\
\hline$h$ & 0,014 & 0,018 \\
& $(0,0036)^{*}$ & $(0,0038)^{*}$ \\
$h^{2}$ & $-0,0015$ & $-0,0020$ \\
$R^{2}$ & $(0,00068)^{*}$ & $(0,0007)^{*}$ \\
$F(2,25)$ & - & - \\
AR(1) & 68,40 & 98,60 \\
AR(2) & $P(0,00)$ & $P(0,00)$ \\
Teste de validade dos instrumentos, & $P(0,00)$ & $P(0,00)$ \\
Hansen-Robusto & $P(0,54)$ & $P(0,54)$ \\
Teste de validade dos instrumentos, & $P(0,999)$ & $P(0,999)$ \\
Sargan & $P(0,722)$ & $\mathrm{P}(0,492)$ \\
Instrumento Adicional & & $n$ \\
$N$ & 416 & 416 \\
\hline
\end{tabular}

* Indica nível de significância de 1\%. Os desvios-padrão entre parênteses são robustos às condições de auto-correlação e heterocedasticidade entre painéis.

A coluna (3) da Tabela 5 reporta ainda a estimativa dinâmica realizada com a variável quantidade de horas trabalhadas semanalmente nos estados como instrumento adicional. Esta variável supostamente captura as diferenças existentes entre os mercados de capital humano dos estados, refletindo não somente as condições de oferta e demanda de capital humano, mas, também, as políticas econômicas de 
cada estado. Como resultado, os coeficientes aumentaram seus valores e se aproximaram ainda mais dos valores obtidos na Tabela 4.

A validade dos resultados do modelo dinâmico da Tabela 5 está subordinada às condições de autorregressividade. As estimativas são válidas neste método, mesmo sob condição de autorregressão de primeira ordem, $\mathrm{AR}(1)$, mas não de segunda, $\mathrm{AR}(2)$. A significância de $\mathrm{AR}(1)$ captura as variáveis omitidas ou informações não observadas. Esta é a grande vantagem destas estimativas dinâmicas, pois são robustas às questões de variáveis omitidas variantes no tempo e/ou efeitos fixos no tempo não observados.

As estatísticas confirmam as condições de efeitos não observados, sendo a probabilidade de não-autocorrelação de primeira ordem igual a zero, e de segunda ordem, equivalente a $54 \%$, portanto, rejeita-se a autocorrelação no $\operatorname{AR}(2)$, confirmando a validade do modelo. Para verificar a qualidade da estabilidade dos resultados obtidos foram realizados dois testes sobre os resíduos. O primeiro de autocorrelação serial dos resíduos proposto por Wooldridge (2001). Nos resíduos da primeira equação, a probabilidade de não-autocorrelação serial foi de $71,04 \%$, enquanto nos resíduos da segunda foi de 70,9\%. A estacionariedade dos resíduos foi testada utilizando o teste de raiz unitária para painéis de dados de Hadri (2000). A estacionariedade dos resíduos foi confirmada sob os aspectos de homocedasticidade e heterocedasticidade. Para o primeiro, a probabilidade foi de $67,7 \%$, e para o segundo, foi de $66,9 \%$ de aceitação da estacionariedade. Estes resultados confirmam a qualidade e estabilidade dos ajustes obtidos pelos modelos dinâmicos.

A validade dos instrumentos foi observada sob dois testes. O teste de Hansen (1982), que é o recomendado para as estimativas realizadas, isto é, estimativas robustas para problemas de heterocedasticidade e autocorrelação entre os painéis, indicou a probabilidade dos instrumentos serem ortogonais de 99,9\%. A outra estatística de validade dos instrumentos de Sargan (1958) reportada, apesar de não ser robusta aos problemas de heterocedasticidade e autocorrelação entre os painéis, confirma a validade dos instrumentos. Ou seja, no conjunto os resultados parecem confirmar a hipótese de que a escolaridade é a causa do crescimento econômico. A instrumentalização desta medida de capital, por ela própria defasada cinco anos, indica o papel da acumulação de capital humano em acordo com preconizado pela equação (6) do modelo?.

O nível de escolaridade que causa o máximo de crescimento econômico é obtido com a derivação parcial da equação estimada na Tabela 4 em relação à variável independente $\mathrm{E}$ :

$$
\begin{aligned}
& \frac{\partial g_{y}}{\partial E}=0.014-0.0030 E=0 \\
& \frac{\partial g_{y}}{\partial E}=0.018-0.0040 E=0
\end{aligned}
$$

\footnotetext{
${ }^{9}$ Foram utilizadas defasagens menores e estas se mostraram insignificantes em seus coeficientes.
} 
De acordo com as equações acima, o nível de escolaridade que produz a taxa máxima de crescimento econômico se situa entre 4,5 e 4,7 anos. Portanto, os estados que estão abaixo deste nível devem privilegiar suas políticas educacionais como forma de obter retornos crescentes em suas taxas de crescimento econômico.

\section{CONCLUSÃO}

Neste artigo foi desenvolvido um modelo teórico que pressupõe o capital humano como causa do crescimento econômico. A explicação adicional do modelo teórico para os efeitos contraditórios - presentes na literatura - entre escolaridade no crescimento econômico, além da não-linearidade, é de duas naturezas: i) o sacrifício inicial de crescimento econômico para que ocorra a acumulação de capital humano, e este ultrapasse a barreira da escolaridade mínima; e, ii) os efeitos quase ínfimos do nível de escolaridade quando esta é bastante elevada. A combinação destas duas informações em uma única base de dados pode gerar resultados contraditórios em estimativas lineares.

A questão da causalidade entre as duas variáveis foi considerada através de estimativas dinâmicas. A partir das estimativas econométricas foi possível verificar que os aumentos nos níveis de escolaridade dos estados geram efeitos em suas taxas de crescimento do PIB per capita, quando se consideram cinco anos de defasagem. Portanto, as políticas de acumulação de capital humano (investimento em educação) são importantes determinantes do crescimento do PIB per capita nos estados brasileiros; tais políticas demonstraram ser robustas mesmo quando controlados os efeitos feedback do próprio crescimento econômico.

A relação não-linear encontrada, na forma de $U$ invertido, entre crescimento econômico e escolaridade, demonstra que o efeito da escolaridade na taxa de crescimento econômico é crescente na primeira fase e decrescente na segunda. O efeito máximo ocorre quando o nível de escolaridade está no intervalo entre 4,5 e 4,7 anos. Este resultado está em acordo com a literatura que preconiza o papel importante exercido pelas instituições, no sentido de que estas passam a exercer um papel mais preponderante na segunda fase, na qual o papel da educação é decrescente diretamente. Ou seja, a educação auxilia diretamente a produtividade na primeira fase e cria mecanismos para que os seus efeitos sejam duradouros na segunda fase, através da criação e consolidação das instituições. Portanto, entendese que esta é a direção na qual as pesquisas devem seguir, no sentido de testar a complementaridade de ambas as políticas.

Para finalizar, deve-se enfatizar que os dados de escolaridade utilizados referem-se à população acima de 25 anos; e que, somado ao efeito de potencial defasagem de cinco anos de escolaridade no crescimento econômico, tem-se como implicação principal o fato de que políticas educacionais devem ser contínuas e de caráter duradouro para se atingir o propósito de uma maior taxa de crescimento per capita no longo prazo. 


\section{REFERÊNCIAS BIBLIOGRÁFICAS}

ARELLANO, M. e BOND, S. R. (1991). "Some tests of specification for panel data: Monte Carlo evidence and an application to employment equations". Review of Economic Studies, 58:277297.

ARELLANO, M. e BOVER, O. (1995). "Another look at the instrumental variable estimation of errorcomponents models". Journal of Econometrics, 68:29-51.

BALTAGI, B. H. (1995). Econometric Analysis of Panel Data. New York: John Willey \& Sons.

BARRO, R. (1990). "Government spending in a simple model of endogenous growth". Journal of Political Economy, 98:103-125.

BARRO, R. J. e SALA-I-MARTIN, X. (1995). Economic Growth. New York: McGraw-Hill.

BALTAGI, B. H. e LI, Q. (1995). "Testing AR(1) against MA(1) disturbances in an error component model”. Journal of Econometrics, 68:133-151.

BENHABIB, J. e SPIEGEL, M. (1994). "The role of human capital in economic development: evidence from aggregate cross-country data”. Journal of Monetary Economics, 34 (2):143-174.

BECK, T., LEVINE, R. e LOAYZA, N. (2000). "Finance and the sources of growth". Journal of Financial Economics, 58(2):261-300.

BILS, M. e KLENOW, P. J. (2000). “Does scholling cause growth?” The American Economic Review, 90(5):1160-1183.

BLUNDELL, R. e BOND, S. (1998). "Initial conditions and moment restrictions in dynamic panel data models". Journal of Econometrics, 87:115-143.

BREUSCH, T. e PAGAN, A. (1980). "The LM test and its applications to model specification in econometrics”. Review of Economic Studies, 47:239-254.

DENISON, E. (1962). The Sources of Economic Growth in the United States. New York: Committee for Economic Development.

DIAS, J. e DIAS, M. H. A. (2004). "Educação dos jovens, analfabetismo e custo do governo: teoria e aplicações econométricas para o Brasil”. Anais do XXII Encontro Nacional de Economia ANPEC, João Pessoa - PB.

DIAS, J. e DIAS, M. H. A. (2007). "Crescimento econômico e as políticas de distribuição de renda e investimento em educação nos estados brasileiros: teoria e análise econométrica”. Estudos Econômicos, 37(4):701-743.

DIAS, J. e MCDERMOTT, J. (2006). “ Education, institutions, and growth: the role of entrepreneurs”. Journal of Development Economics, 80(1):299-328.

EASTERLY, W. (2001). “The lost decades: developing countries' stagnation in spite of policy reform 1980-1998”. Journal of Economic Growth, 6(1):135-157.

EASTERLY, W. E LEVINE, R. (2001). "It's not factor accumulation stylized facts and growth models". The World Bank Economic Review, 15(2):177-219.

GLAESER, E. L., LA PORTA, R., LOPEZ-DE-SILANES, F. e SHLEIFER, A. (2005). "Do institutions cause growth?" Journal of Economic Growth, 9:271-303.

GREENE, W. H. (2000). Econometric Analysis. New Jersey: Prentice Hall.

HALL, R. E. e JONES, C. I. (1999) "Why some countries produce so much more output per worker than others?" The Quarterly Journal of Economics, 114(1):83-117.

HADRI, K. (2000). “Testing for stationarity in heterogeneous panel data”. Econometrics Journal, $3(1): 148-161$.

HANSEN, L. P. (1982). "Large sample properties of generalized method of moments estimators". Econometrica, 50:1029-1054.

HANUSHEK, E. A. e KIMKO, D. D. (2000). "Schooling, labor-force quality, and gowth of nations". American Economic Review, 90(5):1184-208.

HAUSMAN, J. A. (1978). "Specification tests in econometrics”. Econometrica, 46:1251-1272.

IPEA-Instituto de Pesquisas Econômicas e Aplicadas. Indicadores IPEA. Disponível em: <http://www. IPEAdata.gov.br.htm>. 
JUDGE, G. G., GRIFFITHS, W. E., HILL, R. C., LUTKEPOHL, H. e LEE, T. C. (1985). The Theory and Practice of Econometrics. New York: John Wiley and Sons.

KLENOW, P. J. e RODRIGUEZ-CLARE, A. (1997). NBER Macroeconomics Annual. Cambridge: MIT Press.

KRUEGER, A. B.; LINDAHL, M. (2000). “Education for growth: why and for whom?” Journal of Economic Literature, 39(4):1101-1136.

LUCAS, R. (1988). "On the mechanics of economic development". Journal of Monetary Economics, 22:3-42.

MANKIW, G.; ROMER, D.; WEIL, D. (1992). “A contribution to the empirics of economic growth". Quarterly Journal of Economics, 107: 407-437.

REBELO, S. (1991). "Long run policy analysis and long run growth". Journal of Political Economy, 99: 500-521.

ROMER, P. M. (1990). “Endogenous technological change”. Journal of Political Economy, 98: S71102.

SARGAN, J. (1958). "The estimation of economic relationships using instrumental variables". Econometrica, 26(3):393-415.

SCHULTZ, T. W. (1962). "Reflections on investment in man". Journal of Political Economy, 70 (5): 1-8.

UZAWA, H. (1965)."Optimum technical change in an aggregative model of economic growth". American Economic Review, 6(1):18-31.

WOLF, E. N. (2000). "Human capital investment and economic growth: exploring cross country evidence. Structural Change and Economic Dynamics, 11(4):433-472.

WOOLDRIDGE, J. (2002). Econometric Analysis of Cross Section and Panel Data. Cambridge: MIT Press. 